\title{
Journal of Surgery
}

Pattanayak S. J Surg 2017: JSUR-137.

\section{A Case Report of Bone Metastasis from Gastric Cancer}

\author{
Sibaprashad Pattanayak ${ }^{*}$ \\ WHO Fellow in Surgical Oncology, MKCG Medical College, Berhampur, Odisha, India
}

"Corresponding author: Sibaprashad Pattanayak, WHO Fellow in Surgical Oncology, MKCG Medical College, Berhampur, Odisha, India. Tel: +91 9337017800; E- Mail: sibaprashad234@gmail.com

Citation: Pattanayak S (2017) A Case Report of Bone Metastasis from Gastric Cancer. J Surg 2017: JSUR-137. DOI: 10.29011/ JSUR-137.000037

Received Date: 24 April, 2017; Accepted Date: 15 May, 2017; Published Date: 25 May, 2017

\begin{abstract}
Background: Bone metastasis is an uncommon event in metastatic gastric cancer patients and rarely detected as isolated lesions. A case featuring unusual skeletal metastasis of gastric cancer is presented.

Case Report: A 60-year-old man presented with metastasis in the lumber vertebra one and half years after surgery (distal radical gastrectomy for gastric carcinoma). The histopathology of primary tumor was poorly differentiated adenocarcinoma. Patient had received five cycles of adjuvant chemotherapy (Paclitaxel + Carboplatin) before he presented with lumber vertebra metastasis with a seven-month disease free period.

Discussion: The metastatic path of gastric cancer cells is generally haematogenous. Higher incidence of bone metastasis occurs in the axial skeleton. Prognosis of patients exhibiting bone metastases from Gastric cancer is worse compared with other solid tumors.

Conclusion: Skeletal metastasis is an uncommon event in advanced metastatic Gastric cancer though not rare. The clinician must screen the patient for skeletal lesions right from the time of initial diagnosis.
\end{abstract}

\section{Keywords: Gastric Cancer; Metastasis}

\section{Introduction}

Gastric Cancer (GC) is the fourth most common malignancy and the third leading cause of cancer related mortality among males and the fifth leading cause of cancer related mortality among females worldwide, with $\sim 1,000,000$ new patients diagnosed every year [1]. Recurrence of gastric carcinoma most frequently involves abdominal dissemination and this usually occurs within 5 years of primary surgery [2]. Late recurrence is uncommon. Recurrences usually present in the abdominal cavity, peritoneum, lymph nodes, and liver $[3,4]$. Isolated recurrence limited to bone and bone marrow is rare, and prognosis in such cases is poor [5]. We report a rare case of skeletal metastasis in a case of poorly differentiated gastric cancer after adjuvant chemo and a disease-free period of seven months in 1 and half year follow up.

\section{Case Report}

A 60-year-old man presented to the Department of Surgical oncology of MKCG Medical College, Odisha with a 1-month history of progressively increasing lower back pain and pain over the lateral aspect of right lower limb. This patient was operated with a distal radical gastrectomy with D2 lymphadenectomy one and a half year back. The post $\neg$ operative course was normal. Histopathological, it was predominantly a poorly differentiated tubular adenocarcinoma with areas of signet-ring cell carcinoma. There were multiple small volume nodes in the para aortic, peri pancreatic areas.

Post-operatively he was treated with adjuvant chemotherapy with Paclitaxel and Carboplatin. Paclitaxel 175mg/m2IV 3-hour infusion Day 1 and Carboplatin 5 AUC IV infusion for 90 min Day 1. This regimen was repeated every three weeks for five cycles. Patient did not come for sixth cycle. Patient was lost to follow up and came after 8 months with a features of progressive pain lower back over the spine and pain right sciatica in the previous 23 weeks. On clinical examina $\neg$ tion, there was paraparesis with reduced muscle tone and reflexes. Magnetic Resonance Imaging (MRI) of the entire spine revealed numerous metastatic lesions in vertebrae; in particular, the Lumbar (L2 L4) vertebra. Furthermore, several vertebral bodies exhibited marrow infiltration, particularly L2 and L4, and a soft tissue mass was identified in the spinal cord canal from L2 to L4. A CT guided biopsy of the soft tissue at the 
Citation: Pattanayak S (2017) A Case Report of Bone Metastasis from Gastric Cancer. J Surg 2017: JSUR-137.

L2 level revealed metastatic adenocarcinoma with signet ring cells which resembled the histology of the previous gastric cancer. No tumor recurrence was detected in the anastomotic site or elsewhere with upper GI endoscopy, X-ray, CT or MRI examinations. A diagnosis of solitary bone metastasis from gastric carcinoma was therefore made.

\section{Discussion}

While bone metastasis is frequent in patients with breast cancer, lung cancer, renal cancer, prostate cancer, bladder cancer and other primary cancers, it is fairly uncommon inmalignant tumors of the gastrointestinal tract [6-8]. Yoshikawa and Kitaoka [9] reported the incidence of bone metastasis is $1 \sim 20 \%$. In 1987, Nishidoi and Koga [10] have reported that in 246 gastric cancer patients, bone metastasis was associated in 33 patients (13.4\%). Seto, et al. [11] have reported that bone scintigraphy was performed on 60 patients, and bone metastasis was suspected in $25 \%$ of these patients. Bone metastasis may occur more frequently in cases with primary cancer in the body of stomach, poorly differentiated adenocarcinoma and in cases with abundant lymph node metastasis in the vicinity.

Gastric cancer usually metastasizes to the liver, peritoneum, lymph nodes and lungs, whereas bone metastases are uncommon, occurring in $\sim 13.4 \%$ of autopsy cases and are rarely detected as isolated lesions $[6-8,10,12]$. The metastatic path of gastric cancer cells is generally hematogenous through the bone marrow. The gastric mucosa has a rich capillary network and the bone marrow does not contain lymphatic vessels [13].

This hypoth $\neg$ esis is supported by the observation that a higher incidence of bone metastasis occurs in the axial skeleton, such as the spine, pelvic bones or the sternum, where there is a higher content of hematopoietic bone marrow in adults. Therefore, the bone marrow, rather than the bone tissue, is the target in bone metastasis [14]. The prognosis of patients exhibiting bone metastases from Gastric cancers is worse compared with other solid tumors, with a mean survival period of $<5$ months and the longest survival period reported in the literature, 3.5 years [15]. Early detection of bone metastases is difficult as, according to the majority of important international guidelines [16].

In the metastatic setting, treatment aims at symptomatic relief to the patient to improvethe quality of life and prolong symptom free survival. Fluoropyrimidine, taxanes and platinum based regimens are the most commonly used chemotherapeutic approaches, providing response rates of $3050 \%$ and a median overall survival of $\sim 1$ year [17].

\section{Conclusion}

To conclude the prognosis of bone metastasis caused by gastric cancer is very poor and the prognosis of patients may be poorer due to delayed diagnosis and delayed treatment. It seems prudent that tests to assess for bone metastasis need to be done at the time of initial diagnosis and post treatment follow up. Bone scintig- raphy with its high sensitivity appears to be most useful. If such protocols are followed, early diagnosis about skeletal involvement can be made and patients may get potential benefit from radiation and pain relief measures thus improving the quality of life. This requires extensive studies with bigger sample size to evaluate the efficacy of different protocols which may be offered.

\section{References}

1. Jemal A, Bray F, Center MM, Ferlay J, Ward E, et al. (2011) Global cancer statistics. CA Cancer J Clin 61: 69-90.

2. Katai H, Maruyama K, Sasako M, Sano T, Okajima K, et al. (1994) Mode of recurrence after gastric cancer surgery.Dis Surg11: 99-103.

3. Deng J, Liang H, Wang D, Sun D, Pan Y, et al. (2011) Investigations of the recurrence patterns of gastric cancer following curative resection. Surg Today 41: 210-215.

4. Yoo CH, Noh SH, Shin DW, Choi SH, Min JS (2000) Recurrence following curative resection for gastric carcinoma. Br J Surg 87: 236-242.

5. Ahn JB, Ha TK, Kwon SJ (2011) Bone metastasis in gastric cancer patients. J Gastric Cancer 11: 38-45.

6. Crivellari D, Carbone A, Sigon R, Buonadonna A, Cannizzaro R, et al. (1995) Gastric cancer with bone marrow invasion at presentation: case report and review of the literature. Tumori 81: 7476.

7. Noda N, Sano T, Shirao K, Ono H, Katai H, et al. (1996) A case of bone marrow recurrence from gastric carcinoma after a nine year disease free interval. Jpn J ClinOncol 26: 472475.

8. Abrams HL, Spiro R and Goldstein N (1950) Metastases in carcinoma; analysis of 1000 autopsied cases. Cancer 3: 7485.

9. Yoshikawa K, Kitaoka H (1983) Bone metastasis of gastric cancer. Jpn J Surg13: 173-176.

10. Nishidoi H, Koga S (1987) Clinicopathological study of gastric cancer with bone metastasis. Gan To Kagaku Ryoho 14: 17171722.

11. Seto M, Tonami N, Koizumi K, Sui O, Hisada K (1983) [Bone metastasis in gastric cancer-clinical evaluation of bone scintigrams]. Kakulgaku20: 795-801.

12. Moon YW, Jeung HC, Rha SY,Yoo NC, Roh JK, et al. (2007) Changing patterns of prog $\neg$ nosticators during 15 year follow up of advanced gastric cancer after radical gastrectomy and adjuvant chemotherapy: a 15 year follow up study at a single Korean institute. Ann Surg Oncol 14: 27302737

13. Kobayashi M, Okabayashi T, Sano T, Araki K (2005) Metastatic bone cancer as a recurrence of early gastric cancer charac 7 teristics and possible mechanisms. World J Gastroenterol 11: 55875591.

14. Galasko C (1981) The anatomy and pathways of skeletal metastases. In: Bone Metastases. Weiss L, Gilbert A (eds). GK Hall, Boston, MA 1981: 4963.

15. Moon YW, Jeung HC, Rha SY,Yoo NC,Roh JK, et al. (2007) Changing patterns of prog $\urcorner$ nosticators during 15 year follow up of advanced gastric cancer after radical gastrectomy and adjuvant chemotherapy: a 15 year follow up study at a single Korean institute. Ann Surg Oncol 14: 27302737

16. Ajani JA, Bentrem DJ, Besh S, D'Amico TA, Das P, et al. (2013) Gastric cancer, version 2.2013: featured updates to the NCCN Guidelines. J Natl Compr Canc Netw 11: 531546.

17. Field K, Michael M, Leong $T$ (2008) Locally advanced and meta $\neg$ static gastric cancer: current management and new treatment developments. Drugs 68: 299317. 